\title{
Skin decontamination in critically ill patients. Comparison of two daily bathing methods: traditional bath versus $2 \%$ chlorexidine gluconate (CHG) cloths
}

\author{
G Tura $^{1 *}$, N Marcatelli $^{1}, P$ Leardini ${ }^{2}$ \\ From 3rd International Conference on Prevention and Infection Control (ICPIC 2015) \\ Geneva, Switzerland. 16-19 June 2015
}

\section{Introduction}

Healthcare Associated Infections (HAI) in Intensive Care Units (ICU) settings are still a challenge; skin decontamination is a recommended intervention to interrupt pathogen transmission through "source control" ${ }^{\text {. Various studies }}{ }^{1,2,3,4}$ investigated the effectiveness of $2 \%$ CHG cloths compared with the use of traditional bathing (soap and water) or pre - impregnated cloths with soap solution (no antiseptic) for the reduction of MDROs acquisitions and blood-stream infections in Intensive Care Unit settings.

\section{Methods}

The system used for daily patient hygiene at the General Intensive Care Unit (ICU) of the Rimini hospital until April 2013 consisted of a "traditional" method (water and soap). Since May 2013, 2 \% CHG cloths (Sage Products LLC) were introduced for the daily bathing of colonized/infected patients (targeted decolonization) ${ }^{3}$. Afterwards, since October 2013, a new procedure was introduced to extend daily bathing with $2 \%$ CHG cloths to all patients in the ICU (universal decolonization) ${ }^{3}$. Patients between periods had similar characteristics.

\section{Results}

Results compare two periods in which data were collected and analyzed: Period A - November 2012 / April 2013 - 6 months of daily hygienewith soap and water Pre - intervention.
Period B - October 2013 / July 2014 - 10 months of daily hygiene with $2 \%$ CHG cloths (universal decolonization) Intervention. Number of patients Colonized/ Infected: decreased by $51.21 \%(\mathrm{p}$ - value $=0.00141)$. Positive blood cultures decreased by $68.32 \%$ ( $\mathrm{p}$ - value = 0.01699 ) Return on investment(ROI): estimated decrease of $50 \%$ of the total costs, intervention period vs. preintervention period.

\section{Conclusion}

Daily bathing with 2\% CHG cloths significantly reduces the number of patients colonized/infected and MDRO acquisitions. The results and the estimated ROI obtained at the General Intensive Care Unit(ICU) of the Rimini hospital, confirm the opportunity of implementing a universal decolonization protocol in ICU settings.

\section{Disclosure of interest}

None declared.

Authors' details

${ }^{1}$ Rischio Infettivo, Rimini, Italy. ${ }^{2}$ Intensive care unit, Ausl Romagna AOO Rimini, Rimini, Italy.

Published: 16 June 2015

doi:10.1186/2047-2994-4-S1-P239

Cite this article as: Tura et al: Skin decontamination in critically ill patients. Comparison of two daily bathing methods: traditional bath versus $2 \%$ chlorexidine gluconate (CHG) cloths. Antimicrobial Resistance and Infection Control 2015 4(Suppl 1):P239.

${ }^{1}$ Rischio Infettivo, Rimini, Italy

Full list of author information is available at the end of the article 